PROCEEDINGS OF THE

AMERICAN MATHEMATICAL SOCIETY

Volume 127, Number 2, February 1999, Pages 619-625

S 0002-9939(99)04578-5

\title{
GRADIENT ESTIMATES FOR POSITIVE SOLUTIONS OF THE LAPLACIAN WITH DRIFT
}

\author{
BENITO J. GONZÁLEZ AND EMILIO R. NEGRIN
}

(Communicated by Palle E. T. Jorgensen)

\begin{abstract}
Let $M$ be a complete Riemannian manifold of dimension $n$ without boundary and with Ricci curvature bounded below by $-K$, where $K \geq 0$. If $b$ is a vector field such that $\|b\| \leq \gamma$ and $\nabla b \leq K_{*}$ on $M$, for some nonnegative constants $\gamma$ and $K_{*}$, then we show that any positive $\mathcal{C}^{\infty}(M)$ solution of the equation $\Delta u(x)+(b(x) \mid \nabla u(x))=0$ satisfies the estimate

$$
\frac{\|\nabla u\|^{2}}{u^{2}} \leq \frac{n\left(K+K_{*}\right)}{w}+\frac{\gamma^{2}}{w(1-w)},
$$

on $M$, for all $w \in(0,1)$. In particular, for the case when $K=K_{*}=0$, this estimate is advantageous for small values of $\|b\|$ and when $b \equiv 0$ it recovers the celebrated Liouville theorem of Yau (Comm. Pure Appl. Math. 28 (1975), 201-228).
\end{abstract}

\section{INTRODUCTION}

In this paper we investigate the behaviour of positive $\mathcal{C}^{\infty}(M)$ solutions of the equation

$$
\Delta u(x)+(b(x) \mid \nabla u(x))=0
$$

on $M$, where $M$ is an $n$-dimensional complete Riemannian manifold without boundary.

We require smoothness of the manifold, uniform bound on the norm of the vector field $b$ as well as lower bounds on the tensor fields of the Ricci curvature and $\nabla b$ where

$$
\nabla b(X, Y)=\left(\nabla_{X} b \mid Y\right), \quad \forall X, Y \in \mathfrak{X}(M),
$$

where $\mathfrak{X}(M)$ denotes the Lie algebra of vectors fields on $M$ and $\nabla_{X} b$ the associated (Levi-Civita) Riemannian covariant derivative of $b$ with respect to $X$.

Our main result is a gradient estimate for positive $\mathcal{C}^{\infty}(M)$ solutions of equation (1.1), namely,

$$
\frac{\|\nabla u\|^{2}}{u^{2}} \leq \frac{n\left(K+K_{*}\right)}{w}+\frac{\gamma^{2}}{w(1-w)},
$$

Received by the editors May 27, 1997; part of the results of this paper have been presented to Equadiff 95, Lisboa, July 24-29, 1995.

1991 Mathematics Subject Classification. Primary 58G11.

Key words and phrases. Gradient estimate, Laplacian with drift, Bochner-LichnèrowiczWeitzenböck formula, Liouville theorem.

(C)1999 American Mathematical Society 
on $M$, for any $w \in(0,1)$, where the Ricci curvature is bounded below by $-K$, $\nabla b \leq K_{*}$ and $\|b\| \leq \gamma$ for some nonnegative constants $K, K_{*}$ and $\gamma$.

For the particular case when $K=K_{*}=0$ inequality (1.3) yields

$$
\frac{\|\nabla u\|^{2}}{u^{2}} \leq 4 \gamma^{2}
$$

Note that this simple estimation is independent of the dimension of $M$ and for the case when $b \equiv 0$ it recovers the Liouville theorem of Yau [10]. The proof of (1.3), and thus of (1.4), is essentially along the lines of Li and Yau [7] and Davies [3, Chap. 5].

This method, originated first in Yau [10] and Cheng and Yau [2], has been developed by several authors (cf. [3], [6], [7], [8], and [9], amongst others). More specifically, for the case when $b=\nabla \phi$, and $\phi \in \mathcal{C}^{\infty}(M)$, a gradient estimate for any positive $\mathcal{C}^{\infty}(M)$ solution of (1.1) has been obtained by Setti in [9].

In order to start, however, we need an extension of the Bochner-LichnèrowiczWeitzenböck formula for the operator $L^{b}=\Delta+(b \mid \nabla)$. This remarkable fact is proved as an independent lemma. It is known for drift vectors $b=\nabla \phi$ of gradient form; see e.g. the monograph of Deuschel and Stroock (cf. [4], §6.2).

\section{Gradient estimates Revisited}

In the derivation of the main results, a central role will be played by the next formula.

Lemma 2.1 (Bochner-Lichnèrowicz-Weitzenböck formula for $L^{b}$ ). Let $M$ be a Riemannian manifold and assume that $f \in \mathcal{C}^{\infty}(M)$. Then,

$$
L^{b}\left(\|\nabla f\|^{2}\right)=2\|\operatorname{Hess}(f)\|_{H . S .}^{2}+2\left(\nabla f \mid \nabla\left(L^{b} f\right)\right)+2(\operatorname{Ric}-\nabla b)(\nabla f, \nabla f),
$$

where $\|$ Hess $(f) \|_{H . S .}$ denotes the Hilbert-Schmidt norm of Hess $(f)(c f .[4$, p. 262]), Ric denotes the Ricci curvature and $\nabla b$ denotes the tensor field given by (1.2).

Proof. Applying the well-known Bochner-Lichnèrowicz-Weitzenböck formula for the Laplacian, one obtains

$L^{b}\left(\|\nabla f\|^{2}\right)=2\|\operatorname{Hess}(f)\|_{\mathrm{H} . \mathrm{S} .}^{2}+2(\nabla f \mid \nabla(\Delta f))+2 \operatorname{Ric}(\nabla f, \nabla f)+\left(b \mid \nabla\left(\|\nabla f\|^{2}\right)\right)$.

So, in order to prove (2.1) first we establish that

$$
\nabla b(\nabla f, \nabla f)=(\nabla((b \mid \nabla f)) \mid \nabla f)-\frac{1}{2}\left(b \mid \nabla\left(\|\nabla f\|^{2}\right)\right) .
$$

Now, one has

$$
\begin{gathered}
\nabla b(\nabla f, \nabla f)=\left(\nabla_{\nabla f} b \mid \nabla f\right)=\nabla_{\nabla f}((b \mid \nabla f))-\left(b \mid \nabla_{\nabla f} \nabla f\right) \\
=-\left(b \mid \nabla_{\nabla f} \nabla f\right)+\nabla f((b \mid \nabla f))=-\left(b \mid \nabla_{\nabla f} \nabla f\right)+(\nabla((b \mid \nabla f)) \mid \nabla f) .
\end{gathered}
$$

Thus, all that remains is to show that

$$
\nabla_{\nabla f} \nabla f=\frac{1}{2} \nabla\left(\|\nabla f\|^{2}\right) .
$$

In order to check (2.3) let any $Z \in \mathfrak{X}(M)$; then

$$
\begin{gathered}
\left(\nabla\left(\|\nabla f\|^{2}\right), Z\right)=Z\left(\|\nabla f\|^{2}\right)=Z((\nabla f \mid \nabla f)) \\
=2\left(\nabla_{Z} \nabla f \mid \nabla f\right)=2\left(\left(\nabla_{\nabla f} Z+[Z, \nabla f]\right) \mid \nabla f\right) \\
=2\left(\nabla_{\nabla f} Z \mid \nabla f\right)+2([Z, \nabla f] \mid \nabla f)
\end{gathered}
$$




$$
\begin{gathered}
=2\left\{\nabla f((Z \mid \nabla f))-\left(Z \mid \nabla_{\nabla f}\right)\right\}+2([Z, \nabla f] \mid \nabla f) \\
=2\left\{\nabla f(Z f)-\left(Z \mid \nabla_{\nabla f} \nabla f\right)\right\}+2([Z, \nabla f] \mid \nabla f) \\
=2\left\{[\nabla f, Z] f+Z((\nabla f) f)-\left(Z \mid \nabla_{\nabla f}\right)\right\}+2([Z, \nabla f] \mid \nabla f) \\
=-2\left(Z \mid \nabla_{\nabla f} \nabla f\right)+2 Z\left(\|\nabla f\|^{2}\right),
\end{gathered}
$$

where, for any $X, Y \in \mathfrak{X}(M),[X, Y] \equiv X Y-Y X$ is the commutator of $X$ and $Y$. Therefore,

$$
\left(\nabla\left(\|\nabla f\|^{2}\right) \mid Z\right)=-2\left(Z \mid \nabla_{\nabla f} \nabla f\right)+2\left(\nabla\left(\|\nabla f\|^{2}\right) \mid Z\right),
$$

and so

$$
\left(\nabla\left(\|\nabla f\|^{2}\right) \mid Z\right)=2\left(\nabla_{\nabla f} \nabla f \mid Z\right), \quad \forall Z \in \mathfrak{X}(M),
$$

from which (2.3) follows.

Remark 2.1. For the case when $b=-\nabla U, U \in \mathcal{C}^{\infty}(M)$, denoting $L^{U}=L^{-\nabla U}$ and taking into account that $\operatorname{Hess}(U)(X, Y)=\left(\nabla_{X}(\nabla U) \mid Y\right.$ ), for all $X, Y \in \mathfrak{X}(M)$ (cf. $[4$, p. 261]), formula (2.1) is written as

$$
L^{U}\left(\|\nabla f\|^{2}\right)=2\|\operatorname{Hess}(f)\|_{\mathrm{H.S.}}^{2}+2\left(\nabla f \mid \nabla\left(L^{U} f\right)\right)+2(\operatorname{Ric}+\operatorname{Hess}(U))(\nabla f, \nabla f),
$$

which agrees with the Bochner-Lichnèrowicz-Weitzenböck formula derived in $[4, \mathrm{p}$. $262]$ and [5, p. 32].

Now, formula (2.1) enables us to prove the next local gradient estimate.

Theorem 2.1. Let $M$ be a complete Riemannian manifold of dimension n without boundary. Let $B_{p}(2 R)$ be a geodesic ball of radius $2 R$ around $p \in M$ and denote by $-K(2 R)$, with $K(2 R) \geq 0$, a lower bound on $B_{p}(2 R)$ of the Ricci curvature. Set $b$ a vector field on $M$ and denote by $\gamma(2 R)$ and $K_{*}(2 R)$ some nonnegative constants satisfying $\|b\| \leq \gamma(2 R)$ and $\nabla b \leq K_{*}(2 R)$ on $B_{p}(2 R)$, where $\nabla b$ is the tensor field given by (1.2). If $u(x, t)$ is a positive $\mathcal{C}^{\infty}$ solution of the equation

$$
\Delta u(x, t)+(b(x) \mid \nabla u(x, t))=\frac{\partial u(x, t)}{\partial t},
$$

on $M \times[0, \infty)$, then for any $\alpha>1$ and any $w \in(0,1)$, the estimate

$$
\begin{gathered}
\frac{\|\nabla u\|^{2}(x, t)}{u^{2}(x, t)}-\alpha \frac{u_{t}(x, t)}{u(x, t)} \\
\leq \frac{n \alpha^{2}}{2 w t}+\frac{n \alpha^{2}}{2 w}\left\{\frac{2 \epsilon^{2}}{R^{2}}+\frac{(n-1)(1+R \sqrt{K}) \epsilon}{R^{2}}+\frac{\nu}{R^{2}}+\frac{K+K_{*}}{2(\alpha-1)}\right. \\
\left.+\frac{\gamma \epsilon}{R}+\frac{n}{8(1-w)(\alpha-1)}\left(\frac{2 \gamma}{n}+\frac{\alpha \epsilon}{R}\right)^{2}\right\}
\end{gathered}
$$

holds on $B_{p}(R) \times(0, \infty)$, where $\epsilon>0$ and $\nu>0$ are some constants.

Proof. Observe that the function $f(x, t)=\log u(x, t)$ satisfies the equation

$$
L^{b} f+\|\nabla f\|^{2}=f_{t} .
$$

Now, using formula (2.1), it follows that

$$
F(x, t)=t\left\{\|\nabla f\|^{2}(x, t)-\alpha f_{t}(x, t)\right\},
$$


satisfies the estimate

$$
\begin{gathered}
L^{b} F-F_{t}+2(\nabla f \mid \nabla F)+t^{-1} F \\
=t\left[L^{b}\left(\|\nabla f\|^{2}\right)-\alpha L^{b} f_{t}-2\left(\nabla f \mid \nabla f_{t}\right)+\alpha f_{t t}\right. \\
\left.+2\left(\nabla f \mid \nabla\left(\|\nabla f\|^{2}\right)\right)-2 \alpha\left(\nabla f \mid \nabla f_{t}\right)\right] \\
\geq t\left[\frac{2(\Delta f)^{2}}{n}-2\left(K+K_{*}\right)\|\nabla f\|^{2}\right] \\
=t\left[\frac{2}{n}\left\{\|\nabla f\|^{2}+(b \mid \nabla f)-f_{t}\right\}^{2}-2\left(K+K_{*}\right)\|\nabla f\|^{2}\right]
\end{gathered}
$$

on $B_{p}(2 R) \times(0, \infty)$, where we have used the inequalities $\|$ Hess $f \|_{\mathrm{H} . \mathrm{S}}^{2} \geq(\Delta f)^{2} / n$ and $(\mathrm{Ric}-\nabla b) \geq-\left(K+K_{*}\right)$.

Let $\psi$ be a $\mathcal{C}^{\infty}(\mathbb{R})$ function such that

$$
\psi(r)=\left\{\begin{array}{lll}
1 & \text { if } & r \in(-\infty, 1] \\
0 & \text { if } & r \in[2, \infty),
\end{array}\right.
$$

and $0 \leq \psi(r) \leq 1, \forall r \in \mathbb{R}$.

Denote by $\epsilon>0$ and $\nu>0$ some constants with

$$
0 \geq \psi^{-1 / 2}(r) \frac{d}{d r} \psi(r) \geq-\epsilon
$$

and

$$
\frac{d^{2}}{d r^{2}} \psi(r) \geq-\nu
$$

Now we set $\phi(x)=\psi\left(\frac{d(p, x)}{R}\right)$, where $d(p, x)$ is the distance between $p$ and $x$. Using an argument of Calabi [1] (see also Cheng and Yau [2] and Setti [9]), we can assume without loss of generality that the function $\phi$, with support in $B_{p}(2 R)$, is of $\operatorname{class} \mathcal{C}^{2}$.

Let $(a, s)$ be the point in $B_{p}(2 R) \times[0, t]$ at which $\phi F$ takes its maximum value, and assume that this value is positive (otherwise the proof is trivial). Then at $(a, s)$ one has

$$
\nabla(\phi F)=0, \quad \Delta(\phi F) \leq 0, \quad F_{t} \geq 0 .
$$

Therefore at $(a, s)$ one has

$$
\phi \Delta F+F \Delta \phi-2 F\|\nabla \phi\|^{2} \phi^{-1} \leq 0 .
$$

This inequality together with the estimates

$$
\|\nabla \phi\|^{2} \leq \frac{\epsilon^{2} \phi}{R^{2}}
$$

and

$$
\Delta \phi \geq-\frac{(n-1)(1+R \sqrt{K}) \epsilon^{2}}{R^{2}}-\frac{\nu}{R^{2}}
$$

(cf. [1]) yields

$$
\phi \Delta F \leq\left(\frac{2 \epsilon^{2}}{R^{2}}+\frac{(n-1)(1+R \sqrt{K}) \epsilon}{R^{2}}+\frac{\nu}{R^{2}}\right) F, \quad \text { at }(a, s) .
$$

Inequalities (2.6) and (2.7) at $(a, s)$ imply that

$$
\phi \Delta F-(b \mid \nabla \phi) F-\phi F_{t}-2(\nabla f \mid \nabla \phi) F+s^{-1} \phi F
$$




$$
\geq\left\{\frac{2}{n}\left[\|\nabla f\|^{2}+(b \mid \nabla f)-f_{t}\right]^{2}-2\left(K+K_{*}\right)\|\nabla f\|^{2}\right\} s \phi .
$$

From (2.8) the left-hand side of (2.9) satisfies

$$
\begin{gathered}
\phi \Delta F-(b \mid \nabla \phi) F-\phi F_{t}-2(\nabla f \mid \nabla \phi) F+s^{-1} \phi F \\
\leq\left(\frac{2 \epsilon^{2}}{R^{2}}+\frac{(n-1)(1+R \sqrt{K}) \epsilon}{R^{2}}+\frac{\nu}{R^{2}}\right) F-(b \mid \nabla \phi) F-2(\nabla f \mid \nabla \phi) F+s^{-1} \phi F .
\end{gathered}
$$

Denoting $\mu=\frac{\|\nabla f\|^{2}(a, s)}{F(a, s)}$, using (2.9) and the last inequality, we obtain

$$
\begin{gathered}
\left(\frac{2 \epsilon^{2}}{R^{2}}+\frac{(n-1)(1+R \sqrt{K}) \epsilon}{R^{2}}+\frac{\nu}{R^{2}}\right) F \\
+\frac{\epsilon \gamma \phi^{1 / 2} F}{R}+\frac{2(\mu \phi)^{1 / 2} \epsilon F^{3 / 2}}{R}+s^{-1} \phi F \\
\geq\left\{\frac{2}{n}\left(\mu-\frac{\mu s-1}{\alpha s}\right)^{2} F^{2}-\frac{4(\mu F)^{1 / 2} \gamma}{n}\left(\mu-\frac{\mu s-1}{\alpha s}\right) F\right\} s \phi-2\left(K+K_{*}\right) s \mu \phi F .
\end{gathered}
$$

Multiplying this inequality by $s \phi$ and since $\phi^{2} \leq 1$, we obtain

$$
\begin{gathered}
\frac{2(1+(\alpha-1) \mu s)^{2}(\phi F)^{2}}{\alpha^{2} n}-2\left\{\frac{2 s \gamma \mu^{1 / 2}(1+(\alpha-1) \mu s)}{\alpha n}+\frac{\epsilon s \mu^{1 / 2}}{R}\right\}(\phi F)^{3 / 2} \\
-\left\{\left(\frac{2 \epsilon^{2}}{R^{2}}+\frac{(n-1)(1+R \sqrt{K}) R^{2}}{\epsilon}+\frac{\nu}{R^{2}}\right) s+1\right. \\
\left.+\frac{\gamma \epsilon s}{R}+2\left(K+K_{*}\right) \mu s^{2}\right\}(\phi F) \leq 0 .
\end{gathered}
$$

On the other hand, for any $w \in(0,1)$ we have

$$
\begin{gathered}
-2\left\{\frac{2 s \gamma \mu^{1 / 2}(1+(\alpha-1) \mu s)}{\alpha n}+\frac{\epsilon s \mu^{1 / 2}}{R}\right\}(\phi F)^{3 / 2} \\
\geq-\frac{2(1-w)(1+(\alpha-1) \mu s)^{2}(\phi F)^{2}}{\alpha^{2} n}
\end{gathered}
$$

$$
-\frac{n}{2(1-w)(1+(\alpha-1) \mu s)^{2}}\left[\frac{2 s \gamma \mu^{1 / 2}(1+(\alpha-1) \mu s)}{n}+\frac{\epsilon s \alpha \mu^{1 / 2}}{R}\right]^{2}(\phi F) .
$$

From (2.11) inequality (2.10) becomes

$$
A_{1} \lambda^{2}-2 A_{2} \lambda \leq 0
$$

where

$$
\lambda=\phi F, \quad A_{1}=\frac{2 w}{\alpha^{2} n}(1+(\alpha-1) \mu s)^{2},
$$

and

$$
\begin{aligned}
2 A_{2} & =\left(\frac{2 \epsilon^{2}}{R^{2}}+\frac{(n-1)(1+R \sqrt{K}) \epsilon}{R^{2}}+\frac{\nu}{R^{2}}\right) s+1+\frac{\gamma \epsilon s}{R}+2\left(K+K_{*}\right) \mu s^{2} \\
& +\frac{n}{2(1-w)(1+(\alpha-1) \mu s)^{2}}\left[\frac{2 s \gamma \mu^{1 / 2}(1+(\alpha-1) \mu s)}{n}+\frac{\epsilon s \alpha \mu^{1 / 2}}{R}\right]^{2} .
\end{aligned}
$$


As in [3, Lemma 5.3.3], we use the estimate

and so we obtain

$$
\frac{\mu s^{2}}{(1+(\alpha-1) \mu s)^{2}} \leq \frac{s}{4(\alpha-1)},
$$

$$
\begin{aligned}
\frac{2 A_{2}}{A_{1}} \leq \frac{n \alpha^{2}}{2 w}+\frac{n \alpha^{2} s}{2 w}\left\{\left(\frac{2 \epsilon^{2}}{R^{2}}+\frac{(n-1)(1+R \sqrt{K}) \epsilon}{R^{2}}+\frac{\nu}{R^{2}}\right)\right. \\
\left.+\frac{K+K_{*}}{2(\alpha-1)}+\frac{\gamma \epsilon}{R}+\frac{n}{8(1-w)(\alpha-1)}\left(\frac{2 \gamma}{n}+\frac{\epsilon \alpha}{R}\right)^{2}\right\} .
\end{aligned}
$$

Now, since $\lambda \leq 2 A_{2} / A_{1}, s \in[0, t]$ and using (2.12), estimate (2.5) holds.

From Theorem 2.1 one obtains the next global gradient estimate

Corollary 2.1. Let $M$ be a complete Riemannian manifold of dimension n without boundary and assume that the Ricci curvature of $M$ is bounded from below by $-K$ with $K \geq 0$. Also we suppose that the vector field $b$ satisfies $\|b\| \leq \gamma$ and that the tensor field $\nabla b$, given by (1.2), is bounded from above by $K_{*}$, for some nonnegative constants $\gamma$ and $K_{*}$. If $u(x)$ is a positive $\mathcal{C}^{\infty}(M)$ solution of equation (1.1), then for any $w \in(0,1)$, the following estimate holds on $M$ :

$$
\frac{\|\nabla u\|^{2}}{u^{2}} \leq \frac{n\left(K+K_{*}\right)}{w}+\frac{\gamma^{2}}{w(1-w)} .
$$

Proof. Letting $R \rightarrow \infty$ and $t \rightarrow \infty$ in (2.5) one has

$$
\frac{\|\nabla u\|^{2}}{u^{2}} \leq \frac{n \alpha^{2}\left(K+K_{*}\right)}{4 w(\alpha-1)}+\frac{\alpha^{2} \gamma^{2}}{4 w(1-w)(\alpha-1)},
$$

on $M$. Setting $\alpha=2$ (which minimizes the right-hand side of (2.13)), the result holds.

Remark 2.2. If $u(x)$ is a positive $\mathcal{C}^{\infty}(M)$ solution of $\Delta u(x)+(b(x) \mid \nabla u(x))=0$, and assuming that Ric $\geq 0, \nabla b \leq 0$ and $\|b\| \leq \gamma$, for some $\gamma \geq 0$, it follows from Corollary 2.1 above that

$$
\frac{\|\nabla u\|^{2}}{u^{2}} \leq \frac{\gamma^{2}}{w(1-w)},
$$

for any $w \in(0,1)$. Setting $w=1 / 2$ (which minimizes the right-hand side of $(2.14)$ ) one obtains

$$
\frac{\|\nabla u\|^{2}}{u^{2}} \leq 4 \gamma^{2}
$$

Remark 2.3. Let $M=\mathbb{R}$ be the one-dimensional Euclidean space with its standard Riemannian metric. It is a complete Riemannian manifold without boundary and with Ricci curvature identically zero. In this setting consider the equation

$$
u^{\prime \prime}(x)+b u^{\prime}(x)=0
$$

where $b$ is a real constant. It is clear that $u(x)=e^{-b x}$ is a positive $\mathcal{C}^{\infty}(\mathbb{R})$ solution of (2.15), such that $\frac{\|\nabla u\|^{2}}{u^{2}}=b^{2}$. This case is contemplated by Corollary 2.1 with $K=K_{*}=0$ and $\gamma=|b|$, which establishes that $\frac{\|\nabla u\|^{2}}{u^{2}} \leq 4 b^{2}$. 
On the other hand, the equation

$$
u^{\prime \prime}(x)-\left(1+e^{x}\right) u^{\prime}(x)=0
$$

has the function $u(x)=e^{e^{x}}$ as a positive $\mathcal{C}^{\infty}(\mathbb{R})$ solution such that $\frac{\|\nabla u\|^{2}}{u^{2}}$ is unbounded. Note that the function $b(x)=-\left(1+e^{x}\right)$ satisfies $b^{\prime} \leq 0$ and $b$ is unbounded. Thus, we see that the assumption of the boundedness of $\|b\|$ is needed for the kind of results obtained here.

\section{REFERENCES}

[1] E. Calabi, An extension of E. Hopf's maximum principle with an application to Riemannian geometry, Duke Math. J. 25 (1957), 45-56. MR 19:1056e

[2] S.-Y. Cheng and S.-T. Yau, Differential equations on Riemannian manifolds and their geometric applications, Comm. Pure Appl. Math. 28 (1975), 333-354. MR 52:6608

[3] E.B. Davies, Heat Kernels and Spectral Theory, Cambridge Univ. Press, Cambridge, UK, 1990. MR 92a:35035

[4] J.-D. Deuschel and D.W. Stroock, Large Deviations, Academic Press, Boston, 1989. MR 90h:60026

[5] J.-D. Deuschel and D.W. Stroock, Hypercontractivity and Spectral Gap of Symmetric Diffusions with Applications to the Stochastic Ising Models, J. Funct. Anal. 92 (1990), 30-48. MR 91j:58174

[6] J. Li, Gradient estimates and Harnack inequalities for nonlinear parabolic and nonlinear elliptic equations on Riemannian manifolds, J. Funct. Anal. 100 (1991), 233-256. MR 92k:58257

[7] P. Li and S.-T. Yau, On the parabolic kernel of the Schrödinger operator, Acta Math. 156 (1986), 153-201. MR 87f:58156

[8] E.R. Negrin, Gradient estimates and a Liouville type theorem for the Schrödinger operator, J. Funct. Anal. 127 (1995), 198-203. MR 96a:58175

[9] A.G. Setti, Gaussian estimates for the heat kernel of the weighted Laplacian and fractal measures, Canad. J. Math. 44 (5) (1992), 1061-1078. MR 94f:58124

[10] S.-T. Yau, Harmonic functions on complete Riemannian manifolds, Comm. Pure Appl. Math. 28 (1975), 201-228. MR 55:4042

Departamento de Análisis Matemático, Universidad de La Laguna, 38271 Canary IsLANDS, SPAIN

E-mail address: bjglez@ull.es

E-mail address: enegrin@ull.es 